\title{
Experimentelle Untersuchungen an Gasfedern mit einstellbarer Kennlinie für einen Freikolbenmotor
}

\author{
Sven-Erik Pohl $\cdot$ Cornelius Ferrari $\cdot$ Stefan Offinger
}

Eingegangen: 11. September 2010

(C) Springer-Verlag 2010

Zusammenfassung In diesem Artikel werden zwei Gasfedervarianten zur Verwendung in einem Freikolbenmotor experimentell untersucht und bewertet. Die Kennlinien dieser Varianten sind im Betrieb einstellbar. Dabei kann die Gasmasse oder das Gasvolumen verändert werden. Es werden Prüfeinrichtungen vorgestellt, die Parametervariationen an Gasfederprototypen ermöglichen. Die Versuchsergebnisse werden thermodynamisch ausgewertet und verglichen. Es zeigt sich, dass eine massenvariable Gasfeder günstigere thermodynamische Eigenschaften aufweist und daher als Konzept für einen Freikolbenmotor zu bevorzugen ist.

\section{Experiments on Gas Springs with Adjustable Stiffness for a Free-Piston Engine}

\begin{abstract}
In this paper two gas spring designs for a freepiston application are experimentally investigated and evaluated. The free-piston concept demands a gas spring that allows adjustable spring characteristics during operation. Spring characteristics can be changed either by introducing gas to the cylinder or by adjusting the cylinder volume. For both concepts, experimental setups are provided. The results of the experiments are thermodynamically investigated and further compared to find the best gas spring design. The

S.-E. Pohl $(\bowtie) \cdot$ C. Ferrari $~-S$. Offinger

Deutsches Zentrum für Luft- und Raumfahrt (DLR), Institut für

Fahrzeugkonzepte, Pfaffenwaldring 38-40, 70569 Stuttgart,

Deutschland

e-mail: sven.pohl@dlr.de

C. Ferrari

e-mail: cornelius.ferrari@dlr.de

S. Offinger

e-mail: stefan.offinger@dlr.de
\end{abstract}

mass-variable gas spring design can be shown to be preferable over the volume-variable.

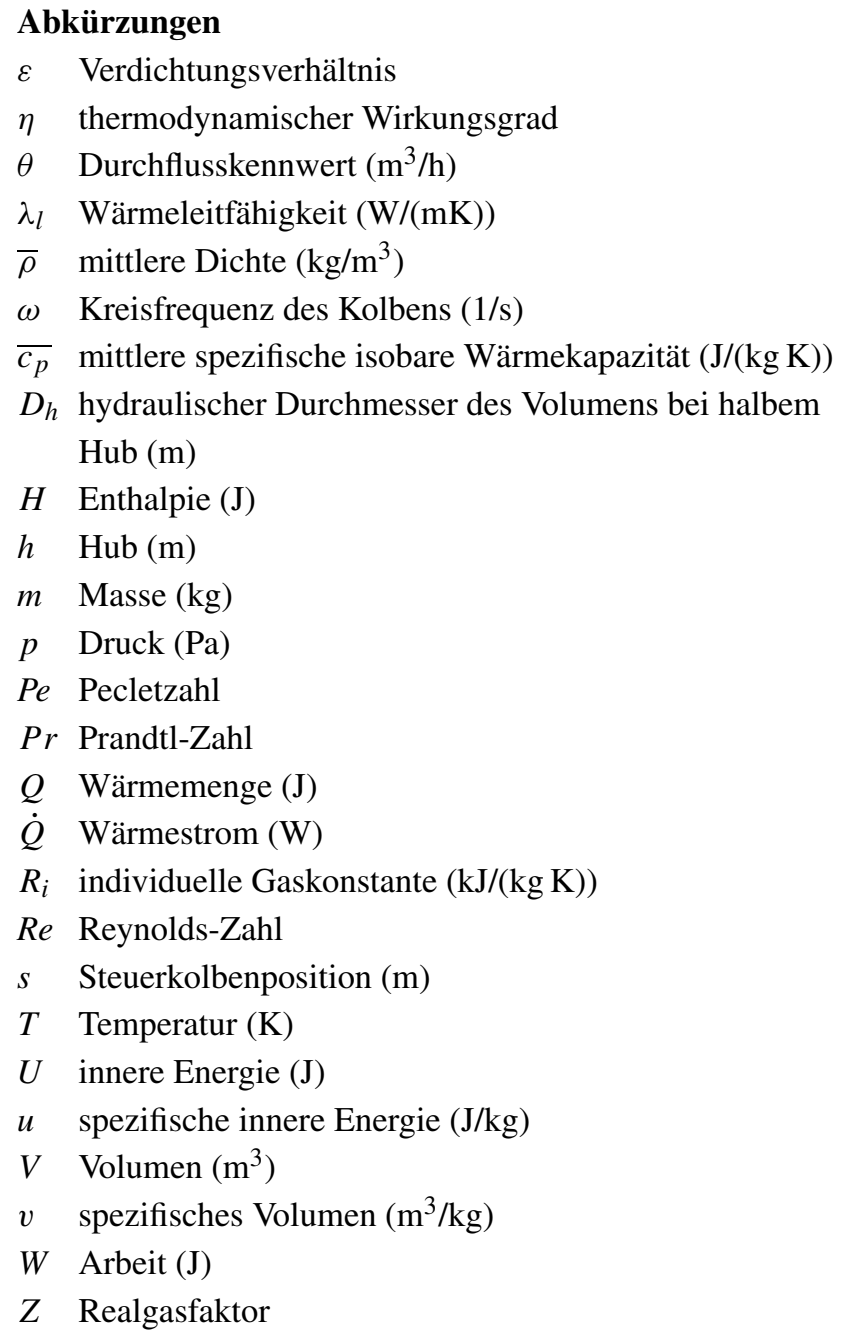




\section{Einleitung}

In Freikolbenmotoren wird die Bewegung des Kolbens direkt in pneumatische, hydraulische oder elektrische Energie umgewandelt. Der frei bewegliche Kolben, der, anders als bei konventionellen Verbrennungsmotoren nicht an einen Kurbeltrieb gekoppelt und dadurch nicht in seiner Bewegung festgelegt ist, ermöglicht neue Freiheitsgrade zur Gestaltung der Verbrennung.

Am Institut für Fahrzeugkonzepte des Deutschen Zentrums für Luft- und Raumfahrt wird ein Freikolbenkonzept zur Auskopplung von elektrischer Energie entwickelt, wobei auf der einen Kolbenseite ein Brennraum und auf der gegenüberliegenden Kolbenseite eine Gasfeder angeordnet ist (Abb. 1). Zwischen den beiden Kolben ist der Läufer des Lineargenerators angebracht, welcher mit Permanentmagneten bestückt ist. Durch den Wegfall der mechanischen Kopplung des Kolbens und den Einsatz einer einstellbaren Gasfeder ist die stufenlose Variation von Hub und Verdichtung möglich. Es wird erwartet, dass solche Freikolbenlineargeneratoren durch die Freiheitsgrade variable Verdichtung und variabler Hub hohe Teillastwirkungsgrade aufweisen und einen effizienten und schadstoffarmen Betrieb mit unterschiedlichen Kraftstoffen ermöglichen.

Als Entwicklungsziel wird eine kompakte Stromerzeugungseinheit mit hohem Wirkungsgrad und reduzierten Emissionen bei geringen Kosten angestrebt. Der Freikolbenlineargenerator ist als Range-Extender Konzept für batterieelektrische Fahrzeuge, als Zusatzstromversorgung (auxiliary power unit, APU) und als Generatoreinheit in einem Blockheizkraftwerk einsetzbar.

Für den energieeffizienten Betrieb des Freikolbenlineargenerators wird eine Gasfeder benötigt, deren Federkennlinie im Betrieb einstellbar ist. Die Gasfeder dient über die Kolbenrückführung hinaus zusätzlich als Energiespeicher, um eine Energieauskopplung durch den Lineargenerator in beiden Bewegungsrichtungen - sowohl Expansion als auch Kompression - gewährleisten zu können. Die damit einhergehende reduzierte Größe des Lineargenerators wirkt sich sowohl positiv auf das Gewicht und die Herstellkosten des Lineargenerators als auch auf das Betriebsverhalten des Gesamtsystems aus.

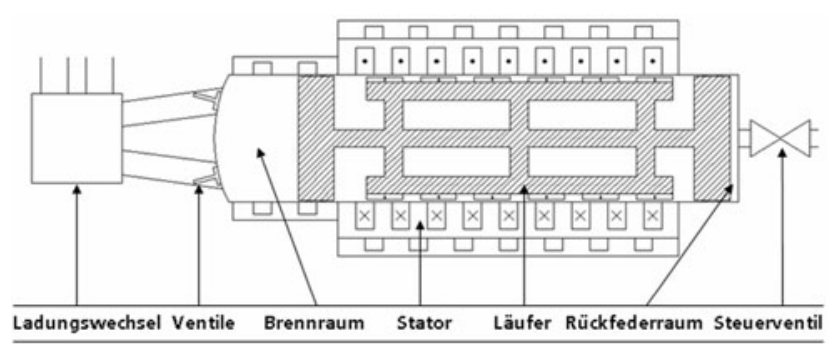

Abb. 1 Schematische Darstellung des DLR-Freikolbenlineargenerators; der Kolben befindet sich verbrennungsseitig im unteren Totpunkt
Gasfedersysteme werden vorwiegend in der Kombination mit Dämpfersystemen, wie zum Beispiel in Federbeinen von Fahrzeugen zur gezielten Dämpfung von eingebrachten Schwingungen eingesetzt. Fundierte Betrachtungen hinsichtlich der Verwendung von Gasfedern in Stirlingmotoren wurden am „Virginia Polytechnic Institute and State University" durchgeführt, deren Ergebnisse in einem Bericht der NASA [5] zusammengefasst sind. Als weitere Beispiele für den Einsatz von Gasfedern können die Kolbenrückführung für Bolzenschubwerkzeuge [8] und die Energiezwischenspeicherung bei der schnellen Bewegungsumkehr von Spundeln in Textilmaschinen [9] genannt werden. Darüber hinaus werden Gasfedersysteme als Kolbenspeicher für Hyraulikanwendungen zur kurzzeitigen Bereitstellung hoher Ölvolumenströme beispielsweise beim hydraulischen Pressen verwendet. Es sind allerdings derzeit keine Gasfederanwendungen bekannt, die eine dynamische Anpassung der Federeigenschaften erfordern, wie sie für den Freikolbenlineargenerator notwendig ist. Als Rahmenbedingungen ergeben sich für den Einsatz in einem Freikolbenlineargenerator Betriebsfrequenzen von bis zu $50 \mathrm{~Hz}$, Hübe von bis zu $100 \mathrm{~mm}$ und Zylinderdrücke von bis zu 50 bar. Es sind daher geeignete Gasfederkonzepte zu entwickeln, die diese Anforderungen erfüllen.

Gasfedern mit im Betrieb einstellbarer Federkennlinie können prinzipiell in zwei Ausführungen realisiert werden. Die Federcharakteristik der Gasfeder können sowohl durch die Änderung der im Zylinder befindlichen Gasmasse (massenvariable Gasfeder) als auch durch Änderung des Gesamtzylindervolumens (volumenvariable Gasfeder) verändert werden [6]. Ausgehend von den theoretischen Betrachtungen in [6] sollen in dieser Veröffentlichung die experimentellen Ergebnisse vorgestellt werden, die an Versuchseinrichtungen für eine massenvariable Gasfeder und eine volumenvariable Gasfeder gewonnen wurden.

\section{Grundlagen}

Zunächst werden die thermodynamischen Vorgänge im Gaszylinder erläutert und Berechnungsgrundlagen sowie Kenngrößen für die Auswertung von Messergebnissen beschrieben.

\subsection{Thermodynamik der Gasfeder}

Die physikalischen Vorgänge in einem Gasfederzylinder sollen im Folgenden durch thermodynamische Bilanzgleichungen betrachtet werden. Das im Zylinder eingeschlossene Gas wird als Phase betrachtet (vgl. [1]), d.h. das Gas besitzt keine inneren Irreversibilitäten, hervorgerufen durch makroskopische Inhomogenitäten in Dichte und Druck. Der Zustand einer fluiden Phase eines reinen Stoffes wird durch 
zwei unabhängige intensive Zustandsgrößen und eine extensive Zustandsgröße festgelegt.

Legt man einen Bilanzraum um das Gas im Zylinder, so kann für die extensive Zustandsgröße $U$ der inneren Energie der 1. Hauptsatz der Thermodynamik in differentieller Form aufgestellt werden:

$\frac{d U}{d t}=\frac{d Q_{i}}{d t}+\frac{d H_{i}}{d t}+\frac{d W_{i}}{d t}$

Hierin ist $U$ die innere Energie des als ortsfest angenommen Gasfederzylinders, $Q_{i}$ die die Systemgrenze überschreitende Wärmemenge, $H_{i}$ die ein- und ausgehende Enthalpie sowie $W_{i}$ die verrichtete Volumenändeurngsarbeit, welche nach (2) definiert ist:

$\frac{d W}{d t}=-p \frac{d V}{d t}$

Neben dem 1. Hauptsatz liefert die Massenbilanz über die ein- und ausströmenden Gasmassen, z.B. durch Ventile oder als Verlustmassenstrom am Kolben vorbei, eine zweite Differentialgleichung:

$\frac{d m}{d t}=\sum \frac{d m_{i}}{d t}$

Des Weiteren werden die thermische Zustandsgleichung und die kalorischen Zustandsgleichung verwendet:

$v(T, p)=\frac{R_{i} \cdot T \cdot Z}{p}$

Darin ist $v$ das spezifische Volumen, $R_{i}$ die individuelle Gaskonstante und $Z$ der Realgasfaktor.

Die kalorische Zustandsgleichung beschreibt die spezifische inneren Energie $u$ des Gases in Abhängigkeit von der Temperatur und dem Druck des Gases:

$u=u(T, p)$

In den (1) bis (5) sind $R_{i}, u$ und $Z$ Funktionen von Druck und Temperatur. Diese Größen werden durch ein Stoffmodel berechnet.

Für die Auswertung der Messdaten, die den Zylinderdruck und das Zylindervolumen umfassen, können die (1) bis (5) herangezogen werden. Der 1. Hauptsatz lässt sich für die Auswertung einer Gasfeder generell schreiben als:

$$
\begin{aligned}
\frac{d U}{d t}= & \frac{d Q_{w}}{d t}+\frac{d H_{V, \text { ein }}}{d t}+\frac{d H_{V, \text { aus }}}{d t} \\
& +\frac{d H_{L, \text { ein }}}{d t}+\frac{d H_{L, a u s}}{d t}-p \frac{d V}{d t}
\end{aligned}
$$

Dabei sind $\frac{d H_{V, \text { ein }}}{d t}$ und $\frac{d H_{V, \text { aus }}}{d t}$ die Enthalpieströme, welche durch das Ventil ein- und ausströmen. $\frac{d H_{L, e i n}}{d t}$ und $\frac{d H_{L, a u s}}{d t}$ sind die Enthalpieströme, welche durch das Blowby am Kolben vorbei aus dem Kontrollvolumen ein- und ausströmen. Die zeitlich aufgelösten Massenströme durch das Ventil und durch den Kolbenspalt (Blowby) werden mit Hilfe der Durchflussgleich nach 7 bestimmt.

$\dot{m}_{e}=\mu A_{2} \sqrt{2 \cdot p_{0} \cdot \rho_{0}}$

$$
\times \sqrt{\frac{\kappa}{\kappa-1}\left(\left(\frac{p_{1}}{p_{0}}\right)^{\frac{2}{\kappa}}-\left(\frac{p_{1}}{p_{0}}\right)^{\frac{\kappa+1}{\kappa}}\right)}
$$

Dabei stellt $\mu$ die Durchflusszahl dar, welche die Reibung und Strahlkontraktion berücksichtigt. $A_{2}$ ist der geometrische Querschnitt in oder nach der Drosselstelle. $\kappa$ ist der Isentropenexponent des Mediums, $p_{0}$ ist der Druck des Mediums vor und $p_{1}$ ist der Druck nach der Drosselstelle. $\rho_{0}$ bezeichnet die Dichte des Mediums vor der Drosselstelle

Bei der Berechnung der zeitlichen Verläufe der Massenströme wird die Durchflusszahl und der geometrische Querschnitt iterativ so lange variiert, bis der Integralwert der ermittelten Massenströme mit den Messwerten, welche nur als Durchschnittswerte vorliegen, übereinstimmen. Die Temperatur des Gases im Zylinder muss mit Hilfe der Druckverlaufsanalyse ermittelt werden. Dabei lassen sich dann auch die spezifischen Enthalpien bestimmen, da der erforderliche Druck messtechnisch erfasst und Temperatur berechnet wird.

Bei der Auswertung der volumenvariablen Gasfeder entfallen die Enthalpieterme, das diese Gasfeder hermetisch dicht ausgeführt ist. Bei dieser Gasfeder wurde Stickstoff als Arbeitsmedium verwendet, so dass das Stoffmodell für Stickstoff nach [3] angesetzt wird. Der 1. Hauptsatz reduziert sich bei dieser Gasfeder auf die Terme Innere Energie, Arbeit und Wandwärme. Die Gasmasse im Zylinder kann mit Hilfe der thermischen Zustandgleichung im Ruhezustand ermittelt werden, da in diesem Zustand neben dem Druck und dem Volumen, welche hochdynamisch messtechnisch erfasst werden können, auch die Temperatur gemessen werden kann. Augrund der Gasdichtheit ändert sich die ermittelte Masse im Betrieb nicht mehr. Somit kann anhand des 1. Hauptsatzes der zeitlich aufgelöste Wandwärmeverlauf eindeutig bestimmt werden.

Bei der Auswertung der massenvariablen Gasfeder kommt den Enthalpieströmen eine große Bedeutung zu. Die Enthalpieströme können als Produkt aus den Massenströmen $\frac{d m}{d t}$ und den spezifischen Enthalpien $h$ berechnet werden. Die zeitlichen Verläufe der Massenströme sind aus der oben dargestellten Iteration zu bestimmen, für die Berechnung der spezifischen Enthalpien und der inneren Energie kommt das Stoffmodell von Zacharias [10] zum Einsatz. Bei der massenvariablen Gasfeder wird Luft als Arbeitsmedium verwendet. Im Gegensatz zu der volumenvariablen Gasfeder muss bei dem massenvariablen System die Anfangstemperatur der Gasmasse im Zylinder geschätzt werden. Es wird mit 
der in $[6,7]$ vorgestellten Simulationsbibliothek eine Starttemperatur im unteren Totpunkt für jeden Betriebspunkt geschätzt

Zur Darstellung der Messergebnisse und zur Bewertung der Gasfederkonzepte werden im Folgenden zwei KenngröBen definiert.

\subsection{Wirkungsgrad einer Gasfeder}

Der thermodynamische Wirkungsgrad eines Gasfedervolumens wird als Verhältnis der mechanischen Arbeit, die bei der Expansion des Gases am Kolben verrichtet wird, zu der mechanischen Arbeit, welcher der Kolben bei der Kompression am Gas verrichtet, definiert:

$\eta_{G F}=\frac{W_{\text {exp }}}{W_{\text {komp }}}=\frac{\int_{V_{O T}}^{V_{U T}} p d V}{\int_{V_{U T}}^{V_{O T}} p d V}$

Sind der Druck $p$ und das Volumen $V$ bekannt, so können die Energieanteile aus der Volumenänderungsarbeit $W=-\int p d V$ ermittelt werden. Bei einer isentropen $\mathrm{Zu}-$ standsänderung beträgt der Wirkungsgrad $100 \%$. In realen Systemen wird dieser Wirkungsgrad allerdings durch Wärme- und Massenverluste reduziert.

$\mathrm{Zu}$ beachten ist, dass bei dieser Wirkungsgraddefinition allein das Gasvolumen betrachtet wird. Die Kolbenreibung der Gasfeder findet keine Berücksichtigung.

\subsection{Pecletzahl für oszillierende Medien}

Zur Auswertung von Messergebnissen kann eine mittlere Pecletzahl [4] berechnet werden, die als charakteristische Größe für den Betriebszustand herangezogen werden kann:

$P e_{\omega}=R e_{\omega} P r=\frac{\bar{\rho}{\overline{c_{p}}} \omega{\overline{D_{h}}}^{2}}{4 \lambda_{l}}$

Darin ist $\bar{\rho}$ die mittlere Dichte pro Kolbenspiel, $\overline{c_{p}}$ die mittlere spezifische isobare Wärmekapazität pro Kolbenspiel, $D_{h}$ der hydraulische Durchmesser des Volumens bei halbem Hub und $\lambda_{l}$ die Wärmeleitfähigkeit des Gases.

Die mittlere Pecletzahl berücksichtigt neben der Gasfedergeometrie und den Gaseigenschaften auch die mittlere Dichte und die Kolbengeschwindigkeit. Wird die mittlere Pecletzahl zur Auswertung von Experimenten auf der Abszisse aufgetragen, so können die Messergebnisse von Parameterstudien differenziert dargestellt werden.

\section{Untersuchungen an einer massenvariablen Gasfeder}

Das Prinzip der massenvariablen Gasfeder beruht auf der Einstellbarkeit der Federsteifigkeit durch Veränderung der

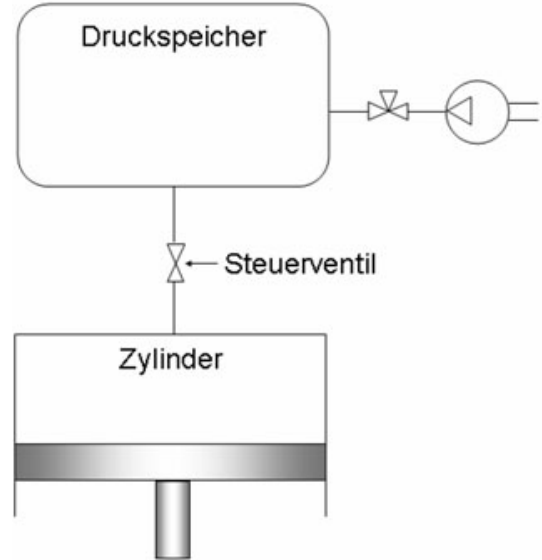

Abb. 2 Funktionsprinzip der Gasmassenanpassung in einer massenvariablen Gasfeder

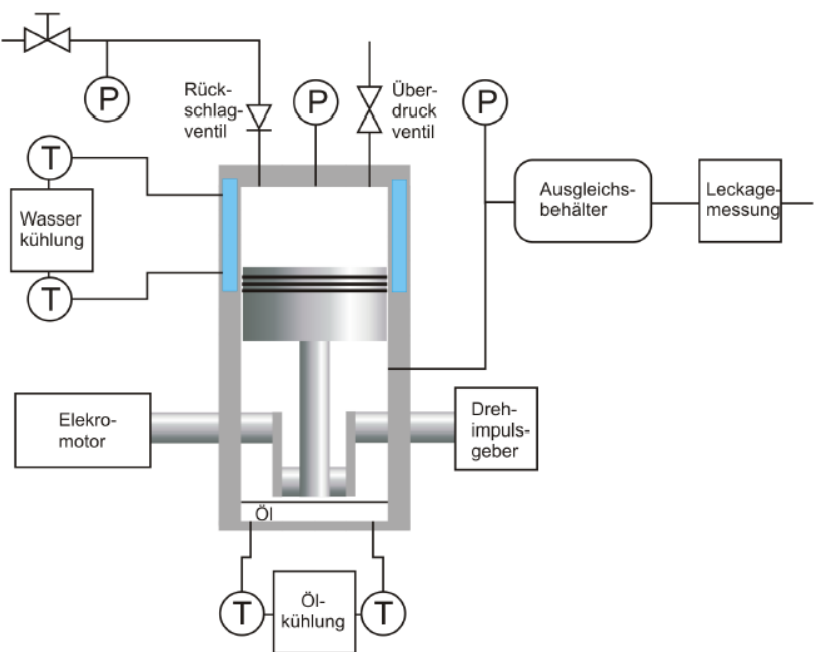

Abb. 3 Prüfstandsaufbau zur Untersuchung einer massenvariablen Gasfeder

Gasmasse im Zylinder. Das Funktionprinzip ist in Abb. 2 dargestellt. Die experimentellen Untersuchungen an einer massenvariablen Gasfeder werden an einem modifizierten Einzylindermotor an einem Motorenprüfstand durchgeführt (Abb. 3).

\subsection{Prüfstandsaufbau}

Für die Untersuchungen an einer massenvariablen Gasfeder steht ein konventioneller Motorenprüfstand zur Verfügung, für den ein Einzylinder-Versuchsmotor aus der Baureihe BMW F-650 modifiziert wird. Der Einzylindermotor bietet sowohl vom Hubvolumen als auch durch seinen modularen Aufbau eine gute Ausgangsbasis für Untersuchungen hinsichtlich einer Gasfederanwendung. Der Motor wird in einem unbefeuerten Zustand betrieben, d.h. die angeschlossene elektromotorische Bremse wird als aktiver Antrieb verwendet. Im ,geschleppten“ Zustand wird im Zylin- 
Abb. 4 Prinzip der Einstellung der Federsteifigkeit durch Anpassung der Gasfedermasse durch ein Ventil

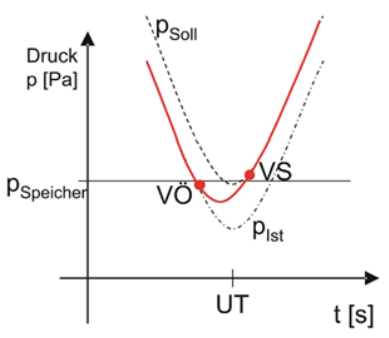

der mit festem Verdichtungsverhältnis Luft komprimiert. In Abb. 3 sind die durchgeführten Modifikationen dargestellt. Es wird ein Zylinderkopf konstruiert, welcher Anschlüsse für ein Überdruckventil und für ein Rückschlagventil vorsieht.

Zur Steuerung der Gasmasse im Zylinder wird ein Rückschlagventil eingesetzt. Unterschreitet der Zylinderdruck den an das Ventil angelegten Vordruck (Abb. 4), so öffnet sich das Ventil während der Niederdruckphase. Ein Druckausgleich ist solange möglich, bis der Zylinderdruck bei der Kompression das Ventil wieder schließt. Über diese sehr einfache Gasmassenregelung kann nicht nur das Druckniveau im Zylinder eingestellt, sondern auch der Verlustmassenstrom ausgeglichen werden, der vorbei an den Kolbenringen in das Kurbelgehäuse strömt.

Der Druck wird im Zylinder, vor dem Rückschlagventil und im Kurbelgehäuse gemessen. Zur Quantifizierung des Leckagemassenstroms wird ein Volumenstrommessgerät verwendet, welches an das Kurbelgehäuse angeschlossen ist.

Ein Drosselventil, das an die zentrale Druckluftversorgung angeschlossen ist, an die steuert den am Rückschlagventil anliegenden Stelldruck. Der Motor besitzt eine Wasserkühlung, die im Standard-Verbrennungsbetrieb auf etwa $80^{\circ} \mathrm{C}$ Vorlauftemperatur eingestellt wird. Der Ölkreislauf wird ebenfalls mit üblichen Parametern betrieben. An der Kurbelwelle des Elektromotors ist eine Drehmesssonde installiert, die die Kurbelposition bereit stellt.

Es werden Versuchsreihen am Motorprüfstand durchgeführt, die eine Druckvariation zwischen 1 und 4 bar im unteren Totpunkt vorsehen, indem der Vordruck entsprechend eingestellt wird. Darüber hinaus wird die Drehzahl zwischen 500 und $3500 \mathrm{U} / \mathrm{min}$ variiert. Abschließend wird die Dynamik einer Vordruckänderung untersucht.

\subsection{Ergebnisse}

Zur Auswertung der Ergebnisse wird in einem ersten Schritt der Wandwärmeverlust berechnet. Dieser ist pro Arbeitsspiel in Abb. 5 über der Pecletzahl und der Drehzahl dargestellt. Der Wandwärmeverlust beträgt abhängig vom Messpunkt zwischen 10 und $30 \mathrm{~J}$ pro Kurbelspiel. Bei konstanter Drehzahl steigt mit wachsendem Mitteldruck auch der Wandwärmeverlust, da mit steigender Druckamplitude

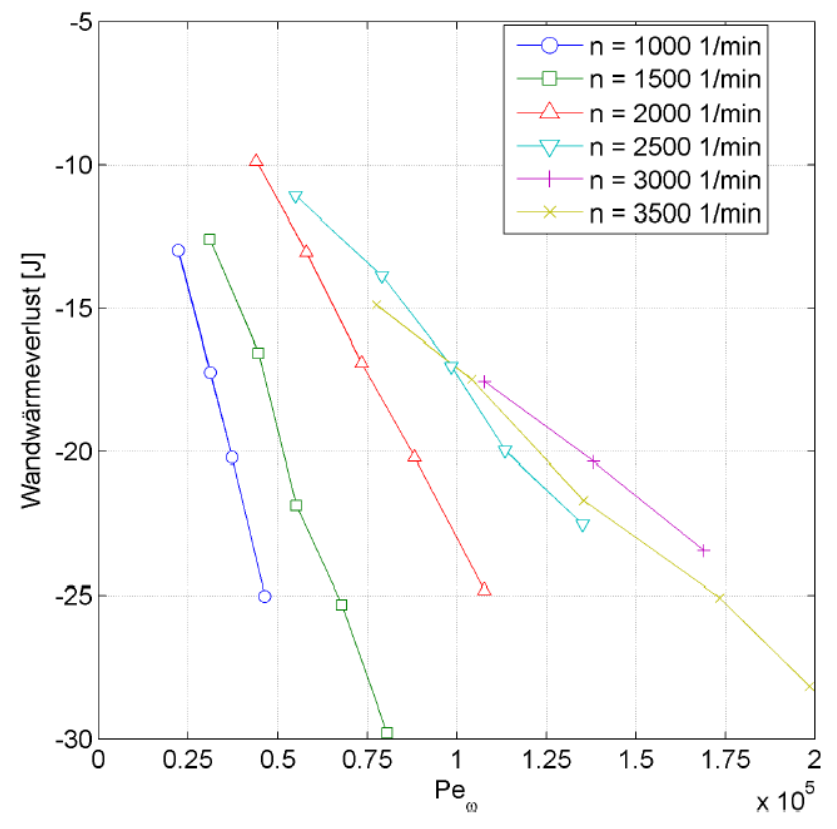

Abb. 5 Wandwärme pro Kolbenspiel für variierende Mitteldrücke und Drehzahlen der massenvariablen Gasfeder

auch die Temperaturschwankung zunimmt und somit der Wandwärmeverlust begünstigt wird. Für ähnliche Mitteldrücke und steigende Drehzahlen bis $2500 \mathrm{U} / \mathrm{min}$ nimmt der Wandwärmeverlust aufgrund der kürzeren Periodendauern ab. Diese Tendenz wird für Drehzahlen von 3000 und $3500 \mathrm{U} / \mathrm{min}$ nicht fortgesetzt, der Wandwärmeverlust wird bei ähnlichen Mitteldrücken größer. Möglicherweise wird durch höhere Turbulenzen in der Grenzschicht des Zylindervolumens der Wärmeübergang begünstigt.

Abbildung 6 zeigt den Wirkungsgrad als Funktion der Pecletzahl für das untersuchte Drehzahlfeld. Aus den Druckmessdaten und der Volumenfunktion können jeweils die Arbeitsanteile für Kompression und Expansion bestimmt werden. Damit liegen die Energieinhalte für die eingespeicherte Energie und die anschließend freigesetzte Energie fest und der Wirkungsgrad kann (8) berechnet werden. Die Wirkungsgrade liegen zwischen $91.6 \%$ und $94.5 \%$. Das gute Ergebnis überrascht angesichts des einfachen Versuchsaufbaus. Für konstante Drehzahlen und bei wachsendem Mitteldruck steigt der Wirkungsgrad tendenziell an. Bis zu einer Drehzahl von $2000 \mathrm{U} / \mathrm{min}$ ist ein Anstieg des Wirkungsgrads zu beobachten, für höhere Drehzahlen ist ein leichter Rückgang zu verzeichnen. Als Ursache können die Beobachtungen zum Wandwärmeverlust angeführt werden, die bei hohen Drehzahlen einen Anstieg des Wandwärmeverlusts erkennen ließen.

Bei einer massenvariablen Gasfeder kommt der Steuerung der Gasmasse eine hohe Bedeutung zu. Für die hier vorgestellten Messungen wurde eine einfache Herangehensweise durch die Verwendung von handelsüblichen Rückschlagventilen gewählt. Im Versuchsbetrieb wurde ein 


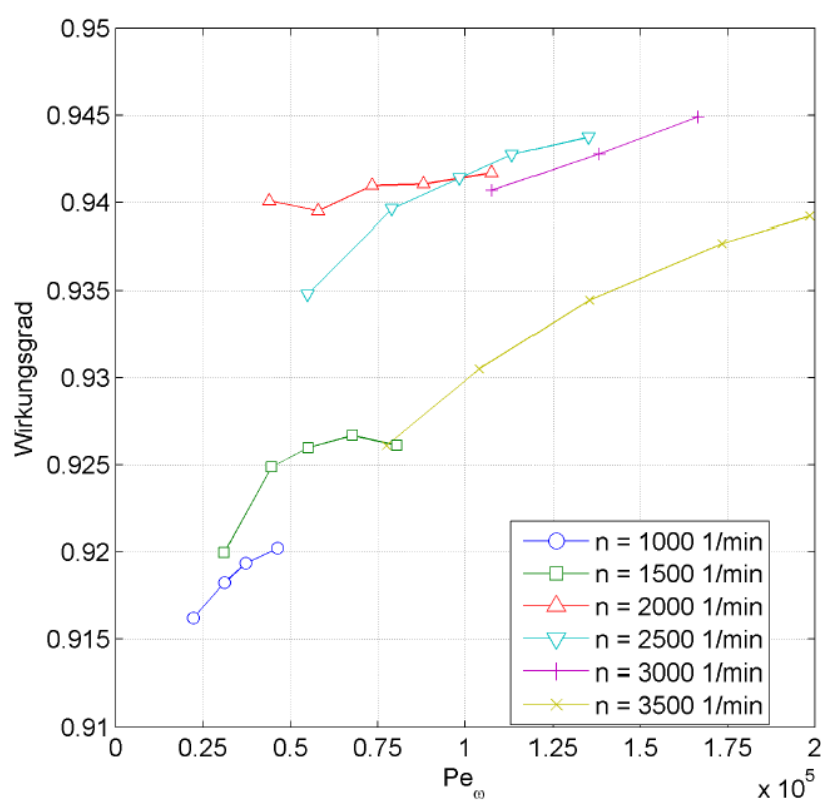

Abb. 6 Wirkungsgrad der massenvariablen Gasfeder für variierende Mitteldrücke und Drehzahlen der massenvariablen Gasfeder

schnelles Versagen des Rückschlagventils beobachtet. Es wird vermutet, dass durch die thermische und mechanische Beanspruchung der schnelle Ausfall begünstigt wurde. Während der Versuchsreihe wurden Rückschlagventile verschiedener Hersteller und mit unterschiedlichen Schaltdrücken untersucht; sie alle führten nach kurzer Zeit zum Defekt. Für weitere Untersuchungen an massenvariablen Gasfedern sind daher hydraulisch oder elektromagnetisch betriebene Tellerventile zu bevorzugen, die die notwendige Kombination aus niedrigen Schaltzeiten, hohen Spitzendrücken und hohen Durchflüssen realisieren können.

Des Weiteren ist zu beobachten, dass sich eine Druckdifferenz zwischen dem Vordruck und dem tatsächlichen Druck im unteren Totpunkt einstellt. Dieser Druckunterschied lässt darauf schließen, dass der Volumenstrom durch das Rückschlagventil zu gering ist, um in der aktiven Zeitspanne, in der das Ventil geöffnet ist, den Druck auszugleichen. Ein Ventil mit einer größeren effektiven Fläche kann hier Verbesserung bringen.

\section{Untersuchungen an einer volumenvariablen Gasfeder}

Für die Untersuchungen an einer volumenvariablen Gasfeder wird eine Konstruktion gewählt, die eine gasdichte Betreiben der Gasfeder ermöglicht. Dadurch ist die Gasmasse im Zylinder zu jedem Zeitpunkt konstant und es muss während des Betriebs keine Masse über ein hochdynamisches Ventil nachgeführt werden. Um dennoch eine Einstellbarkeit der Federsteifigkeit im Betrieb zu gewährleisten, wird ein zweiter im Betrieb verstellbarer Kolben vorgesehen, mit dem das Gasfedervolumen angepasst werden

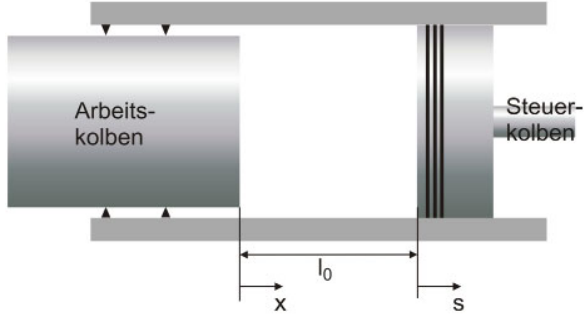

Abb. 7 Prinzipskizze der volumenvariable Gasfeder

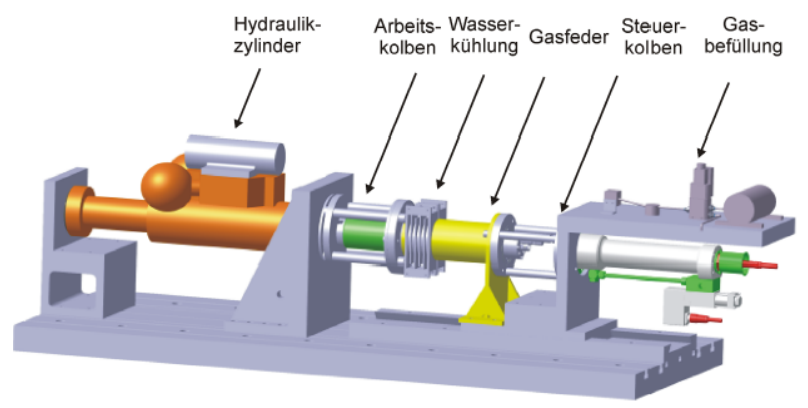

Abb. 8 Prüfstandsaufbau zur Untersuchung einer volumenvariablen Gasfeder

kann. Die Prinzipskizze einer volumenvariablen Gasfeder ist in Abb. 7 dargestellt.

\subsection{Prüfstandsaufbau}

Es wird eine Gasfeder entworfen, die über zwei Kolben verfügt: den Arbeitskolben, der für eine hohe Dynamik ausgelegt wurde, und einen Steuerkolben, der das Gasfedervolumen einstellen kann. Auf einer Länge von 150 mm können beide Kolben beliebig in einem Stahlzylinder verfahren werden (siehe Abb. 7).

Die Abdichtung des Arbeitskolbens wird durch die Verwendung eines Tauchkolbens realisiert. Der Tauchkolben wird durch ein Zylinderrohr bewegt, in dem zwischen zwei Polymer-Dichtringen ein Ölumlauf für die Schmierung und die Gasdichtung sorgt. Das umströmende Öl dient darüber hinaus zum Abführen der Reibwärme.

Der Steuerkolben wird im Vergleich zum Arbeitskolben nur langsam bewegt und ist daher konventionell mit einer Polymerdichtung versehen. Die Gasbefüllung sowie die Druck- und Temperaturmessung werden im Kolbenboden des Steuerkolbens eingebaut.

Im Vorfeld der Gasfederuntersuchungen wird neben der Gasfeder eine Prüfumgebung konzipiert und aufgebaut, die den Prüfträger hinsichtlich des Einsatzes in einem Freikolbenlineargenerator geeigneten Prüfzyklen unterziehen kann. Diese Prüfumgebung soll beliebige Kolbenbewegungen sowie die freie Einstellbarkeit von Hub und Verdichtung während des Betriebs ermöglichen. 
Aufgrund dieser Anforderungen wird ein Hydraulikzylinder in Verbindung mit einer leistungsfähigen Zentralhydraulikversorgung ausgewählt. Der Hydraulikzylinder wird mit einem Servoventil gesteuert, welches für Volumenströme bis 1000 1/min und einem Hydraulikdruck bis 290 bar ausgelegt ist. An die Kolbenstange des Hydraulikzylinders wird der Arbeitskolben der Gasfeder angeschlossen. Der Kolben kann durch das Hydrauliksystem Kräfte von bis zu $60 \mathrm{kN}$ aufbringen. Es können Hübe von $100 \mathrm{~mm}$ bei Frequenzen von bis zu $25 \mathrm{~Hz}$ realisiert werden.

Ein solches dynamisches Hydrauliksystem kann im Rahmen der technischen Randbedingungen beliebige Wegverläufe abbilden. Dafür wird eine wegbasierte Regelung implementiert, welche das Positionssignal des Kolbens zurückführt und mit dem vorgegebenen Sollsignal vergleicht. Als Stellgröße wird das Positionssignal des Servoventils ausgegeben, mit dem der Hydraulikzylinder angesteuert wird. Aufgrund der geforderten hohen Dynamik war es notwendig die Regelung durch eine Vorsteuerung zu ergänzen. Mit diesem System ist es möglich, den Wegvorgaben sehr genau zu folgen und damit unterschiedliche Kolbenverläufe am Prüfstand zu realisieren.

Der Hydraulikzylinder wird auf einem luftgedämpften Spanntisch an einem Stahlwinkel montiert. An diesem Hauptwinkel wird auch der Gasfederzylinder befestigt. Der Stützfuß der Gasfeder wird auf einem Schwalbenschwanzschlitten befestigt, so dass diese zur Montage des Gasfederzylinders verschoben werden kann. Auf dem Schlitten ist an der Steuerkolbenseite der Gasfeder ein zweiter, kleinerer Hydraulikzylinder montiert, der zur Einstellung des Steuerkolbens vorgesehen ist.

Ein Vorteil der hermetisch dichten, volumenvariablen Gasfederbauweise gegenüber dem leckagebehafteten System der massenvariablen Gasfeder ist die konstante Zylindermasse, die neben der einfacheren Auswertung auch die Gasmasse als Variationsparameter sinnvoll erscheinen lässt. Zur Bestimmung der Gasmasse werden nach Abschluss der Befüllung bei ruhenden Kolben der Zylinderdruck sowie die Gastemperatur gemessen und über das momentane Volumen des Zylinders auf die Gasmasse geschlossen.

Unter Berücksichtung der Rahmenbedingungen lässt sich eine Versuchsmatrix aufstellen, wie sie in Tab. 1 aufgeführt ist. Es entstehen neun Versuchsreihen, die jeweils Frequenzvariationen von $1-20 \mathrm{~Hz}$ vorsehen.

\subsection{Ergebnisse}

Der aus den Messdaten berechnete Wandwärmeverlust für ein Kolbenspiel ist in Abb. 9 über der Pecletzahl dargestellt. Es ergibt sich ein nahezu linearer Zusammenhang zwischen der Pecletzahl und dem Wandwärmeverlust. Sind Frequenz und Gasmasse bei kleinen Pecletzahlen niedrig, so ist auch der Wandwärmeverlust gering. Entsprechend
Tab. 1 Kennfeld der Parametervariation der volumenvariablen Gasfeder

\begin{tabular}{|c|c|c|c|c|c|}
\hline \multirow{2}{*}{$\begin{array}{l}\text { Versuchs- } \\
\text { reihe }\end{array}$} & \multicolumn{2}{|c|}{ Arbeitskolben } & \multirow{2}{*}{$\begin{array}{l}\text { Steuerkolben } \\
s \\
{[\mathrm{~m}]}\end{array}$} & \multirow{2}{*}{$\begin{array}{l}\text { Gasmasse } \\
m_{G} \\
{[\mathrm{~g}]}\end{array}$} & \multirow{2}{*}{$\begin{array}{l}\text { Frequenz } \\
f \\
{[\mathrm{~Hz}]}\end{array}$} \\
\hline & $\begin{array}{l}x_{0} \\
{[\mathrm{~m}]}\end{array}$ & $\begin{array}{l}x_{h} \\
{[\mathrm{~m}]}\end{array}$ & & & \\
\hline 1 & 0.000 & 0.090 & 0.000 & 0.9 & $1,5,10,15,20$ \\
\hline 2 & 0.000 & 0.090 & 0.000 & 3.1 & $1,5,10,15,20$ \\
\hline 3 & 0.000 & 0.090 & 0.000 & 3.9 & $1,5,10,15,20$ \\
\hline 4 & 0.000 & 0.090 & 0.000 & 5.4 & $1,5,10,15,20$ \\
\hline 5 & 0.000 & 0.068 & 0.025 & 0.9 & $1,5,10,15,20$ \\
\hline 6 & 0.000 & 0.068 & 0.025 & 3.1 & $1,5,10,15,20$ \\
\hline 7 & 0.000 & 0.068 & 0.025 & 3.9 & $1,5,10,15,20$ \\
\hline 8 & 0.000 & 0.068 & 0.025 & 5.4 & $1,5,10,15,20$ \\
\hline 9 & 0.000 & 0.046 & 0.050 & 0.9 & $1,5,10,15,20$ \\
\hline 10 & 0.000 & 0.046 & 0.050 & 3.1 & $1,5,10,15,20$ \\
\hline 11 & 0.000 & 0.046 & 0.050 & 3.9 & $1,5,10,15,20$ \\
\hline 12 & 0.000 & 0.046 & 0.050 & 5.4 & $1,5,10,15,20$ \\
\hline
\end{tabular}

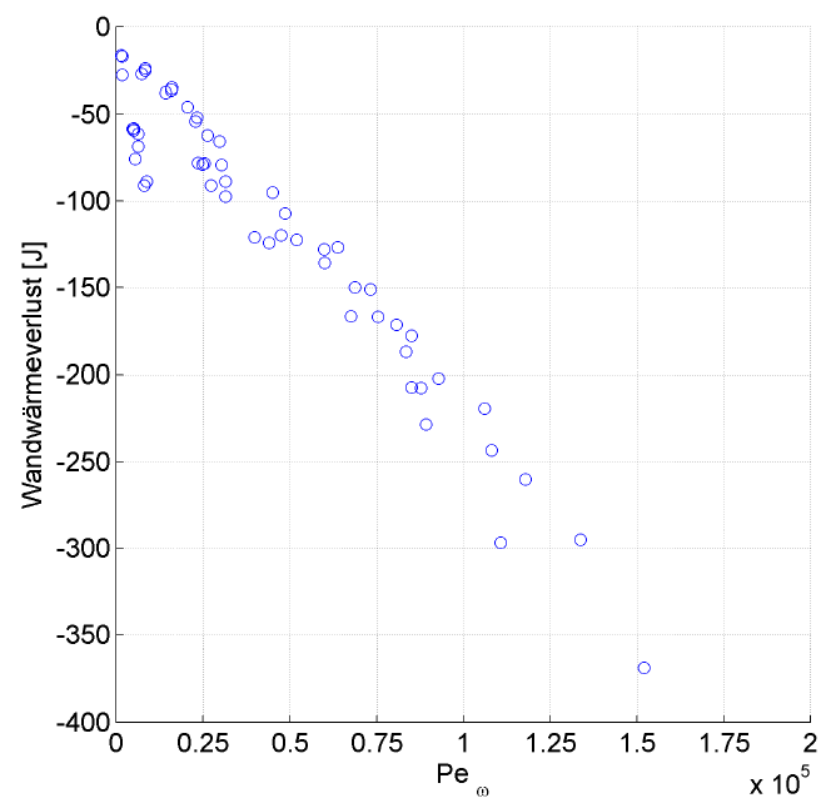

Abb. 9 Wandwärme pro Kolbenspiel für die volumenvariable Gasfeder

steigt der Wandwärmeverlust für höhere Pecletzahlen. Im Vergleich zu den Ergebnissen der massenvariablen Gasfeder liegt der Wandwärmeverlust bei der volumenvariablen Gasfeder um den Faktor zehn höher.

Beeinflusst wird der Wandwärmeverlust maßgeblich durch die Wärmeübertragungsfläche. Aufgrund der Tauchkolbenkonstruktion der volumenvariablen Gasfeder entsteht eine größere Wandfläche als beispielsweise bei einer massenvariablen Gasfeder, wie Abb. 10 zeigt. Während die Wärmeübertragungsfläche einer massenvariablen Gasfeder bei 

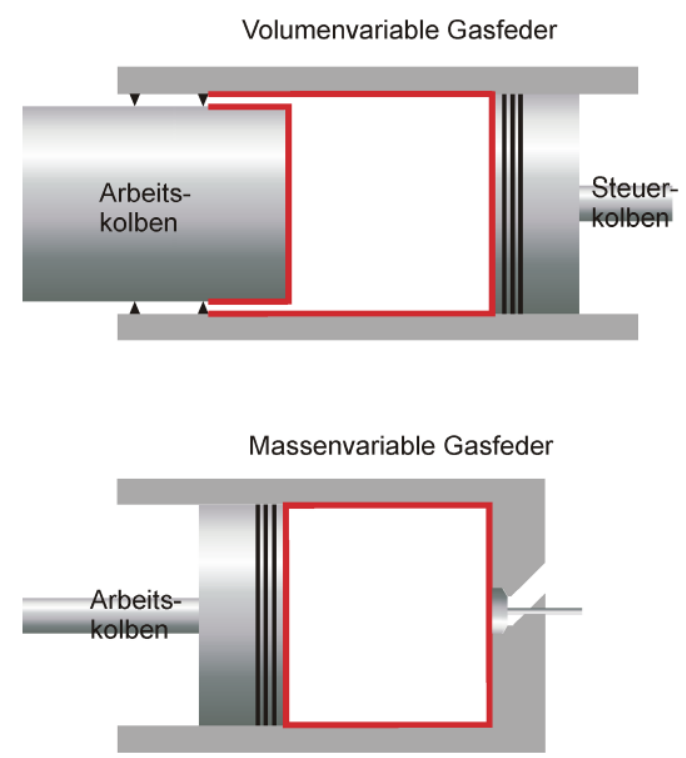

Abb. 10 Vergleich der Wärmeübertragungsfläche einer massenvariablen und einer volumenvariablen Gasfeder

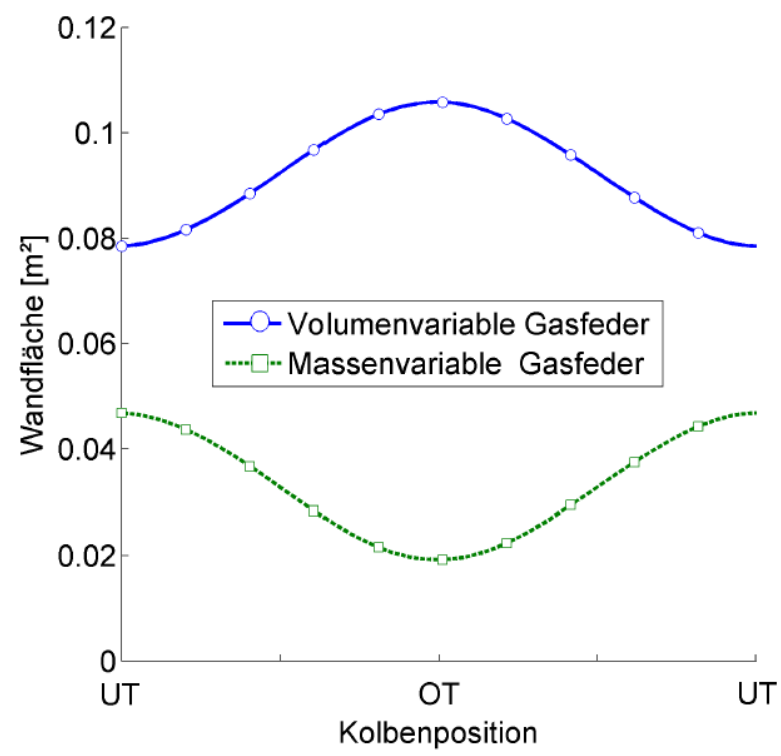

Abb. 11 Verlauf der Wärmeübertragungsflächen bei einem Hub von $h=0.09 \mathrm{~m}$ und der Steuerkolbenposition $s=0 \mathrm{~m}$

der Kompression abnimmt, steigt die Wandfläche durch Eintreten des Tauchkolbens im Falle der volumenvariablen Gasfeder sogar an. Dieser Zusammenhang ist für einen Hub von $h=0.09 \mathrm{~m}$ des Arbeitskolbens und der Steuerkolbenposition $s=0 \mathrm{~m}$ in Abb. 11 dargestellt. Durch die Verwendung eines Tauchkolbens wird die Wärmeübertragungsfläche gerade dann maximal, wenn die Gastemperatur durch Kompression im oberen Totpunkt ihren Höhepunkt erreicht und somit der Wandwärmeübergang begünstigt wird. Der vergleichsweise hohe Betrag des Wandwärmeverlustes in Abb. 9 ist

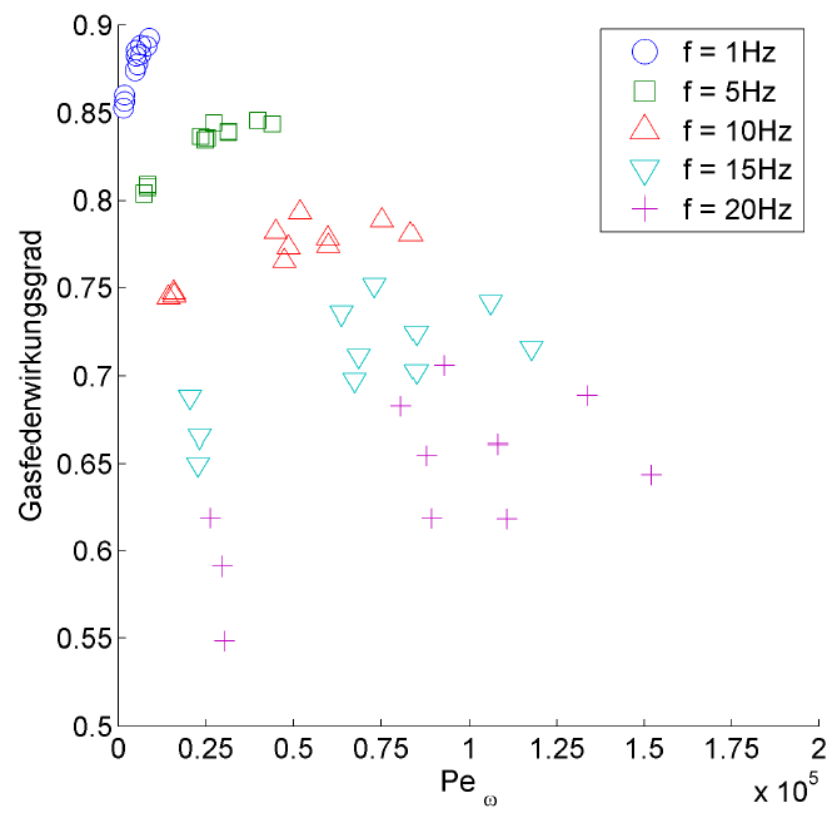

Abb. 12 Wirkungsgrad für variierende Frequenzen der volumenvariable Gasfeder

auf den verstärkenden Effekt des Wärmeübergangs durch den Tauchkolben zurückzuführen.

Für die Darstellung des Wirkungsgrades wird als Abszisse die dimensionslose Pecletzahl aus Abschn. 2.3 verwendet. Der Wirkungsgrad der volumenvariablen Gasfeder liegt zwischen 85 und $90 \%$ für niedrige Pecletzahlen und fällt mit steigender Pecletzahl auf 55 bis $65 \%$ (siehe Abb. 12).

Die Frequenzabhängigkeit des Wirkungsgrades ist in Abb. 12 dargestellt. Es ist zu erkennen, dass der Gasfederwirkungsgrad mit zunehmender Frequenz fällt. Diese Beobachtung steht im Gegensatz zur Wirkungsgradentwicklung bei der massenvariablen Gasfeder.

Zur Erklärung dieser Beobachtung soll zunächst der Temperaturverlauf über einem Arbeitsspiel betrachtet werden. In Abb. 13 ist der Gastemperaturverlauf für Frequenzen von 1 bis $20 \mathrm{~Hz}$ aufgetragen. Dabei ist zu beobachten, dass sich das Maximum der Gastemperatur mit steigender Frequenz vom oberen Totpunkt der Kolbenbewegung entfernt, wie es auch von Bargende [2] am unbefeuerten Motor beschrieben wurde. Allerdings ist die in dieser Arbeit beobachtete Phasenverschiebung deutlich höher. Die Abhängigkeit der Phasenverschiebung der Maximaltemperatur von der Frequenz ist in Abb. 14 dargestellt.

Die berechnete Phasenlage ist für alle Frequenzen negativ, da jeweils das Temperaturmaximum durchlaufen wird, bevor der Kolben den oberen Totpunkt erreicht.

Betrachtet man unter diesem Gesichtspunkt die Energiebilanz des 1. Hauptsatzes aus (1) und setzt sie in die Definition des Gasfederwirkungsgrades aus (8) ein, so entsteht ein Ausdruck für den Wirkungsgrad, der in Abhängigkeit vom 


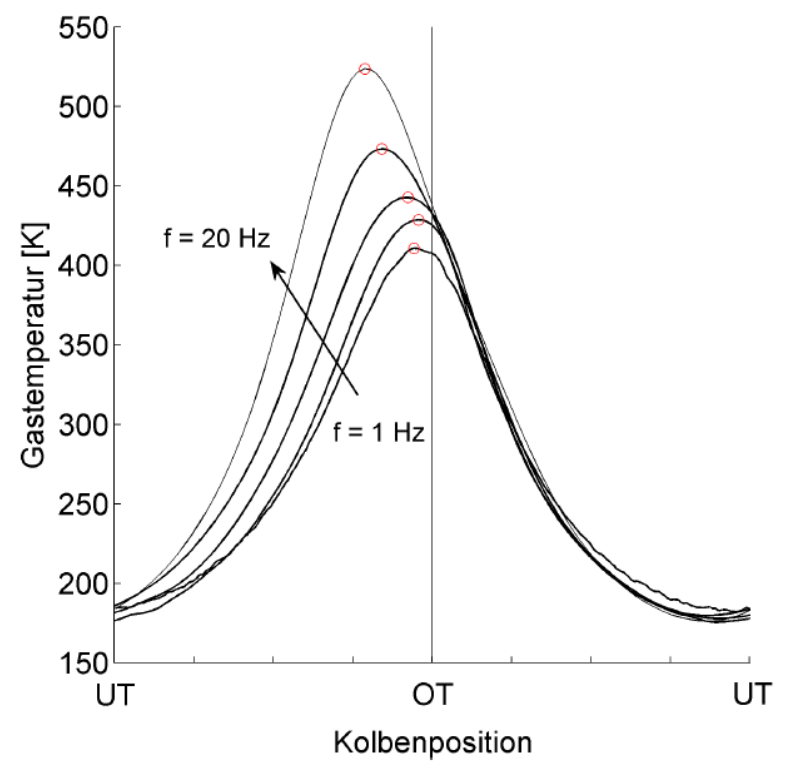

Abb. 13 Verlauf der Gastemperatur über einer Periode für unterschiedliche Frequenzen

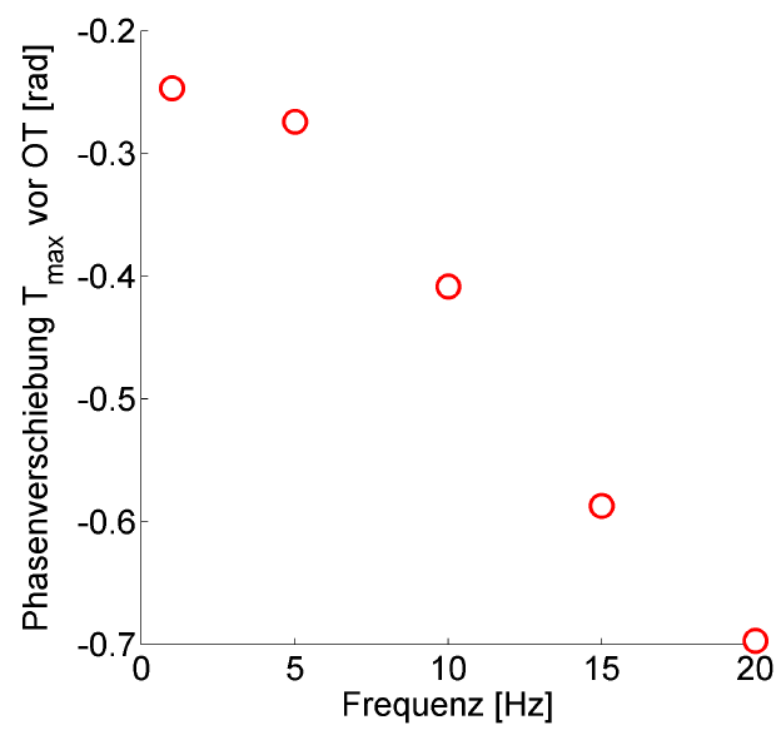

Abb. 14 Phasenverschiebung des Temperaturmaximums in Abhängigkeit von der Frequenz

Wandwärmestrom $\dot{Q}$ und der spezifischen, inneren Energie $u$ formuliert ist:

$\eta=\frac{\int_{V_{O T}}^{V_{U T}} p d V}{\int_{V_{U T}}^{V_{O T}} p d V}=\frac{\int_{t_{O T}}^{t_{U T}} \dot{Q} d t+\int_{u_{O T}}^{u_{U T}} m d u}{\int_{t_{U T}}^{t_{O T}} \dot{Q} d t+\int_{u_{U T}}^{u_{O T}} m d u}$.

Sowohl der Wandwärmestrom als auch die innere Energie sind abhängig von der Gastemperatur. Eine Verschiebung des Temperaturmaximums bei steigender Frequenz in Richtung der Kompressionsphase hat daher einen Anstieg des Nenners in (10) zur Folge, so dass bei steigender Frequenz der Wirkungsgrad der Gasfeder sinkt. Da die
Verschiebung des Wärmeübergangsschwerpunktes bei den Messungen zur massenvariablen Gasfeder in diesem Maße nicht aufgetreten ist, wird angenommen, dass dieser Effekt vorwiegend auf die Konstruktion der volumenvariablen Gasfeder zurückzuführen ist und kann darauf hindeuten, dass eigentlich ein konjugiertes Problem mit Wandkopplung gerechnet werden müsste, um die Speicherkapazität der Wand mit zu berücksichtigen.

\section{Experimenteller Vergleich der Gasfederkonzepte}

Der Prüfstand für die Untersuchung einer volumenvariablen Gasfeder eignet sich für vergleichende Experimente von massenvariabler und volumenvariabler Gasfeder. Dadurch wird ein direkter Vergleich der zwei Konzepte am identischen Prüfträger möglich. Für den Vergleich der Gasfedervarianten wurden zwei Versuchsreihen durchgeführt. Zunächst wurde eine Messreihe zur volumenvariablen Gasfeder und anschließend eine vergleichende Messung zur massenvariablen Gasfeder aufgenommen. Analog zu dem Parameterkennfeld in Tab. 1 wurde für eine Gasfedermasse von $m_{G}=3.1 \mathrm{~g}$ eine Variation des Kolbenhubes durchgeführt, wobei die Steuerkolbenposition so angepasst wurde, dass das Verdichtungsverhältnis konstant bei $\varepsilon=7.4$ liegt. Die Parameter sind in Tab. 2 zusammengefasst.

Für den Vergleich dieser Messdaten mit einer massenvariablen Gasfeder wird die gleiche gespeicherte Energie sowie der gleiche Kolbenhub der Gasfeder als Kriterium definiert. In einer zweiten Messreihe wurde daher bei gleichem Hub die Gasmasse in der Gasfeder so lange angepasst, bis die gleiche Arbeit bei der Kompression verrichtet wird.

Das Ergebnis des experimentellen Vergleichs der Gasfedervarianten ist in Abb. 15 durch Kraftverläufe über dem Kolbenhub dargestellt. Dabei wird die reine Gaskraft verwendet; die Kolbenreibung wird nicht berücksichtigt. Für den größten Hub sind sowohl für die massenvariable als auch für die volumenvariable Gasfeder die Versuche identisch. Für die Untersuchungen bei kleineren Hüben wurde bei der volumenvariablen Variante der Steuerkolben nachgeführt, bis das angestrebte Verdichtungsverhältnis erreicht wurde. Die Gasmasse ist mit $3.1 \mathrm{~g}$ konstant und die Gaskraft steigt auf etwa $27 \mathrm{kN}$ an. Durch Anpassen der Gasmasse auf $5.8 \mathrm{~g}$ (siehe Tab. 2) wurde dann bei deaktiviertem Steuerkolben $(s=0 \mathrm{~m})$ der Vergleichsversuch zur massenvariablen Gasfeder durchgeführt. Abbildung 15 zeigt einen aufgrund des geringeren Verdichtungsverhältnisses flacheren Kraftverlauf. Zwar liegt zu Beginn der Kompression durch die größere Gasmasse im Zylinder eine höhere Kraft als bei der volumenvariablen Gasfeder vor, jedoch steigt die Kraft im Verlauf der Kompression weniger stark an und erreicht ein Maximum im oberen Totpunkt der Kolbenbewegung von $16 \mathrm{kN}$. Ein dritter Betriebspunkt bei weiter verringertem Kolbenhub zeigt ein ähnliches Bild: während die 
Tab. 2 Parameter und Ergebnisse für den experimentellen Vergleich zwischen massenvariabler und volumenvariabler Gasfeder

\begin{tabular}{lllllllll}
\hline Bezeichnung & & Einheit & \multicolumn{2}{l}{ Massenvar. Gasfeder } & \multicolumn{2}{l}{ Volumenvar. Gasfeder } \\
\hline Arbeitskolben UT & $x_{0}$ & $\mathrm{~m}$ & 0 & 0 & 0 & 0 & 0 & 0 \\
Arbeitskolben Hub & $h$ & $\mathrm{~m}$ & 0.090 & 0.068 & 0.046 & 0.090 & 0.068 & 0.046 \\
Steuerkolben Position & $s$ & $\mathrm{~m}$ & 0 & 0 & 0 & 0 & -0.025 & -0.050 \\
Gasmasse & $m_{\text {gas }}$ & $\mathrm{g}$ & 3.1 & 5.8 & 9.8 & 3.1 & 3.1 & 3.1 \\
Frequenz & $f$ & $\mathrm{~Hz}$ & 1 & 1 & 1 & 1 & 1 & 1 \\
Verdichtungsverhältnis & $\varepsilon$ & - & 7.4 & 2.8 & 1.8 & 7.4 & 7.4 & 7.4 \\
Kompressionsarbeit & $W_{\text {in }}$ & $\mathrm{J}$ & 501 & 504 & 498 & 501 & 501 & 475 \\
Wirkungsgrad & $\eta$ & - & 0.89 & 0.96 & 0.98 & 0.89 & 0.88 & 0.87 \\
\hline
\end{tabular}

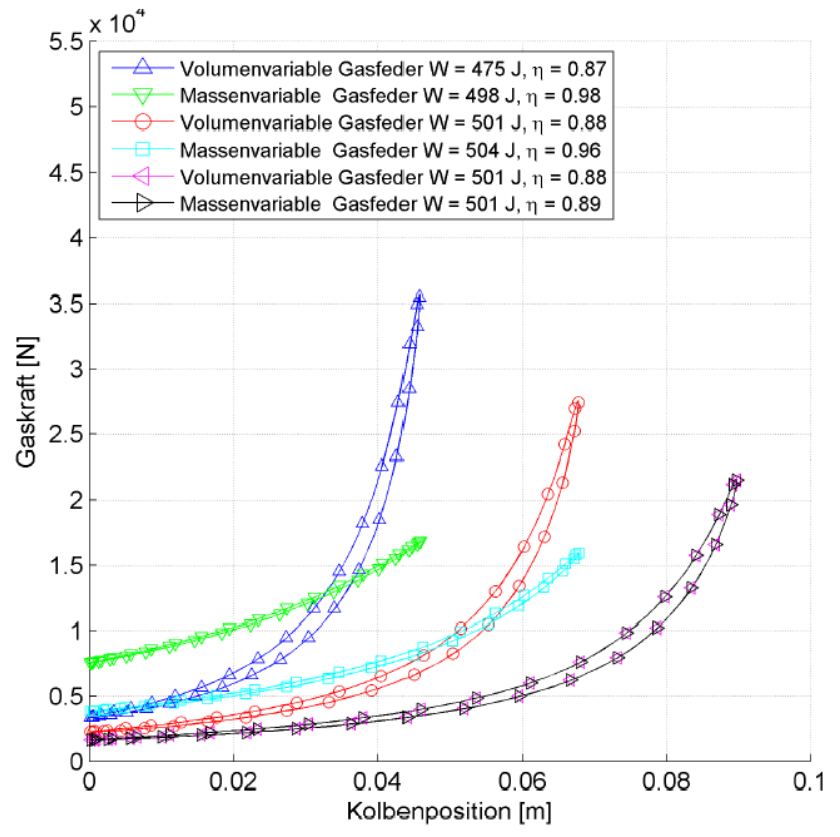

Abb. 15 Experimenteller Vergleich von massenvariabler und volumenvariabler Gasfeder für drei Betriebspunkte

Gaskraft der volumenvariablen Gasfeder auf bis zu $36 \mathrm{kN}$ ansteigt, erreicht die massenvariable Vergleichsmessung eine moderate Kraft von $17 \mathrm{kN}$.

Die Auswirkungen des unterschiedlichen Betriebsverhaltens der zwei Gasfedervarianten auf den Wirkungsgrad lassen sich bereits am Kraftverlauf in Abb. 15 erkennen. Während der Kraftverlauf für die massenvariable Gasfeder für Kompression und Expansion nahezu identisch ist, liegt der Kurvenverlauf für die volumenvariable Gasfeder bei der Expansion unter dem der Kompression. Der Wirkungsgrad der massenvariablen Gasfeder muss dadurch deutlich über dem der volumenvariablen Gasfeder liegen. Die Auswertung der Messergebnisse bestätigt die Beobachtung (vgl. Tab. 2). Während der Wirkungsgrad der massenvariablen Gasfeder mit zunehmend reduziertem Hub von $89 \%$ auf $98 \%$ ansteigt, bleibt er bei der volumenvariablen Gasfeder nahezu konstant. Zu erklären ist dieser Unterschied durch den
Wandwärmeverlust, der durch hohe Zylinderdrücke und dadurch bedingte hohe Temperaturen ansteigt. Für die massenvariable Gasfeder ergibt sich aufgrund des geringeren Mitteldrucks somit ein besser Wirkungsgrad bei den Betriebspunkten mit reduziertem Hub.

\section{Zusammenfassung}

In diesem Artikel wurden zwei Gasfederkonzepte für den Einsatz in einem Freikolbenmotor experimentell untersucht und bewertet. Die für die Verwendung der Gasfedern in einem Freikolbenlineargenerator notwendige Einstellbarkeit der Federkennlinien im Betrieb wird dabei entweder durch eine Veränderung der Gasmasse oder durch das Einstellen des Gasfedervolumens erreicht.

Die massenvariable Gasfeder wurde auf einem Einzylindermotorenprüfstand untersucht. Für die massenvariable Gasfeder konnten geringe Wandwärmeverluste und damit Wirkungsgrade zwischen 92 und $94.5 \%$ erzielt werden. Besonderes Augenmerk muss bei dieser Variante auf die Ventiltechnik gelegt werden. Die verwendeten Rückschlagventile führten schon nach kurzer Zeit zum Defekt. Daher muss für zukünftige Konzepte die Entwicklung schnellschaltender elektromagnetischer Ventile vorangetrieben werden.

Für die Untersuchungen an einer volumenvariablen Gasfeder wurde eine Prüfumgebung realisiert, die durch einen Hydraulikzylinder beliebige Kolbenbewegungen ermöglicht. Die verwendete volumenvariable Gasfeder verfügt neben dem Arbeitskolben über einen verstellbaren Steuerkolben, der im Betrieb das Gesamtvolumen der Gasfeder einstellen kann. Die notwendige Gasdichtheit der volumenvariablen Gasfeder konnte nachgewiesen werden. Bei einem Zylinderdruck von 16 bar beträgt der Druckverlust nur wenige Millibar pro Stunde. Allerdings führt die aufwendige Abdichtung zu einer hohen Kolbenreibung. Darüber hinaus konnten bei den Messungen hohe Wandwärmeverluste und ein mit steigender Frequenz fallender Wirkungsgrad beobachtet werden, der auf die verwendete Tauchkolbenkonstruktion zurückzuführen ist. Dieser Tauchkolben führt auf- 
grund seiner aussenliegenden Dichtung bei steigender Verdichtung zur Vergrößerung der Wandfläche, während bei der massenvariablen Gasfeder die Wandfläche abnimmt.

Auch im direkten Vergleich beider Gasfederkonzepte im Prototyp der volumenvariablen Gasfeder, bei dem konstruktive Unterschiede eliminiert werden, führt das Konzept der massenvariablen Gasfeder zu höheren Wirkungsgraden. Dieser Umstand ist auf die niedriegeren Mitteldrücke bei gleicher eingespeicherter Energie beim massenvariablen Konzept zurückzuführen.

Es konnte mit den experimentellen Untersuchungen gezeigt werden, dass die massenvariable Gasfeder das thermodynamisch günstigere Konzept für die Verwendung in einem Freikolbenlineargenerator darstellt. Zukünftige Entwicklungen werden sich dadurch auf das massenvariable Konzept konzentrieren. Dabei kommt der Entwicklung eines hochdynamischen Gasfederventils eine besondere Bedeutung zu.

\section{Literatur}

1. Baehr HD, Kabelac S (2006) Thermodynamik - Eine Einführung in die Grundlagen und ihre technischen Anwendungen, 13 Aufl. Springer, Berlin
2. Bargende M (1991) Ein Gleichungsansatz zur Berechnung der instationären Wandwärmeverluste im Hochdruckteil von Ottomotoren. Dissertation, Technische Hochschule Darmstadt

3. Elsner N (1982) Grundlagen der Technischen Thermodynamik, 3 Aufl. Akademie-Verlag, Berlin

4. Kornhauser AA (1989) Gas-wall heat transfer during compression and expansion. Dissertation, Massachusetts Institute of Technology

5. Kornhauser AA, Kafka BC, Finkenbeiner DL, Cantelmi FC (1994) Heat transfer measurements for stirling machine cylinders. Technischer Bericht NAG3-1285, Virginia Polytechnic Institute and State University

6. Pohl S-E, Ferrari C (2007) Vergleich von Gasfedervarianten mit variabler Kennlinie für einen Freikolbenmotor. Forschung im Ingenieurwesen 71(3-4):181-188

7. Pohl S-E, Gräf M (2005) Dynamic simulation of a free-piston linear alternator in modelica. In: Schmitz G (Hrsg) Modelica 2005. Hamburg

8. Rullmann SH (1998) Entwicklung einer Gasfeder als Kolbenrückführung für Bolzenschubwerkzeuge. Dissertation, RWTH

9. Spörri M (2004) Bewegungsdynamik und Verlustminimierung bei schneller Bewegungsumkehr mit pneumatischer Energiezwischenspeicherung. Dissertation, ETH Zürich

10. Zacharias F (1970) Mollier-I,S-Diagramme für Verbrennungsgase in der Datenverarbeitung. MTZ 31(7):296-303 